\title{
Elderly vaccination-The glass is half full
}

\author{
Chandini Raina MacIntyre \\ School of Public Health and Community Medicine, University of New South Wales, Sydney, Australia; \\ r.macintyre@unsw.edu.au
}

Received 22 October 2013; revised 25 November 2013; accepted 1 December 2013

Copyright (C) 2013 Chandini Raina MacIntyre. This is an open access article distributed under the Creative Commons Attribution License, which permits unrestricted use, distribution, and reproduction in any medium, provided the original work is properly cited.

\begin{abstract}
The shifting global demography and ageing of populations worldwide brings with new challenges for the health care, and an imperative for healthy ageing and preventive health strategies for adults. Immunization is the low hanging fruit for healthy ageing, and yet under-utilized for various reasons, including waning immunity in the elderly, lack of RCT data in this age group, and lack of provider confidence in vaccines for the elderly. The elderly people have a higher incidence of infection and more severe and serious consequences of infection. Diseases such as influenza, pneumococcal disease and herpes zoster have long been recognized as causing a high burden in the elderly, but evidence is also emerging for other infections such as pertussis being a major cause of the morbidity in this age group. Now, there are several vaccines which can prevent major infectious diseases in the elderly. To improve uptake of these vaccines, elderly vaccination should be viewed through a different lens to pediatric vaccination, accepting that vaccines are less immunogenic in the elderly. The population health impact of vaccines in the elderly, despite of immunosenescence and lower immunogenicity, is still likely to be high given the increased disease incidence. Vaccination is an important and readily available means of prevention in the elderly.
\end{abstract}

Keywords: Health Ageing; Immunization; Vaccination; Elderly

\section{AGEING AND PREVENTION OF INFECTION}

The median age of population of the world is forecasted to increase from 26.6 years in 2000 to 45.6 years in 2100, affecting all regions of the globe [1]. In devel- oped countries, it is predicted that by the middle of this century, $25 \%$ of the population will be aged $>65$ years, and further, the proportion of elderly people in residential aged care facilities will increase substantially [2,3]. This shifting demography brings with it new challenges for the health care, and an imperative for healthy ageing and preventive health strategies for adults [4].

The elderly have a higher incidence of infection and more severe and serious consequences of infection [5]. Diseases such as influenza, pneumococcal disease and herpes zoster have long been recognized as causing a high burden in the elderly, but evidence is also emerging for other infections such as pertussis being a major cause of the morbidity in this age group [6]. In most countries, acute hospital care consumes $>90 \%$ of the health budget. Pneumonia not only results in hospital admissions of the elderly, but also results in a significant cost to primary care [7]. There is also evidence that the influenza vaccination prevents the acute myocardial infarction, with infection being a likely trigger for the acute thrombosis in underlying diseased arteries [8-10]. It has also been shown that $10 \%$ of AMI patients have undiagnosed influenza, and patients at risk of heart disease are under-vaccinated [8]. There are many opportunities for the prevention of disease through the vaccination for the elderly, and ensuring that vaccine-preventable causes of hospitalization in the elderly are prevented by the appropriate vaccination should be a priority. With the changing demography of society, "grandparent care" of infants and young children is becoming common. Over $29 \%$ of working mothers have a grandparent provide childcare for children $<5$ years of age; and $35.2 \%(432 / 1229)$ of children aged $0-3$ years receive the grandparent care, thus increasing the risk of the infectious disease transmission between the very young and the old [11,12].

Furthermore, the ageing population will necessitate increasing reliance on the elderly for workforce, with likely older retirement ages. The ageing of populations and the consequent increased burden on acute health services will increase the drivers for the primary preven- 
tion and positive ageing in the coming decades. Immunisation is the low hanging fruit. Rapid developments in available vaccines, vaccination technology and modes of delivery will offer new opportunities for the prevention in the elderly.

However, challenges to immunisation of the elderly include poor and waning immunity; more difficult access and service delivery issues for immunisation compared to infants; and lack of provider confidence in vaccines for the elderly. Residential aged care has its own special challenges, with outbreaks of gastroenteritis, influenza and other respiratory infections being common and often explosive [13-15]. Outbreaks can be particularly devastating in residential aged care, with high rates of morbidity and mortality, as well as sickness and absenteeism of staff and forced closure of aged care facilities during outbreaks. It has been shown that even an incompletely matched influenza vaccine provides protection during an aged care outbreak [16]. Pertussis is usually thought of as being a serious infection only in infants. However, in addition to influenza and other respiratory viruses, outbreaks of pertussis, including pertussis deaths, occur in the elderly in residential aged care [17-21]. This highlights that pertussis prevention is important in the elderly, not only to prevent transmission to vulnerable infants, but also to protect the elderly themselves from serious morbidity and mortality.

Vaccination rates of the elderly in residential aged care are not always adequate, with the common misconception that pneumonia is “the old man's friend". Most national recommendations for elderly vaccination include influenza and pneumococcal vaccine, yet the frail elderly are less likely to be vaccinated than healthy elderly. It has been shown that demented frail elderly are significantly less likely to be vaccinated than non-demented, and patients $>80$ years are less likely to be vaccinated than those under 80 years [22]. Such subjective judgements by providers disregard the human suffering caused by infectious diseases as well as the fact that communicable diseases impact more than the individual and can cause outbreaks. In addition, demented and frail elderly are frequently admitted to hospital with pneumonia, even if unvaccinated.

\section{AGEING, IMMUNITY AND EVIDENCE}

Ageing is accompanied by a wide range of defects of both cellular and humoral immunity, termed "immunosenesence" $[23,24]$. Reduced numbers and defects in antigen-presenting cells such as plasmacytoid dendritic cells have been described; altered activation of $\mathrm{T}$ cells, especially CD8 cells, results in impaired cytokine production and cell mediated immunity; defects in humoral immunity have also been described, with reduced function and diversity of $\mathrm{B}$ cells $[25,26]$. The exponential rise of herpes zoster incidence after the age of 50, as first described by Hope-Simpson, is thought to reflect the corresponding decline in cell-mediated immunity, with the elderly being at risk for herpes zoster despite commonly having high antibody levels to varicella-zoster virus $[27,28]$. Immunosenescence combined with the tendency for waning vaccine-induced immunity for many vaccines of importance to the elderly, such as influenza, pneumococcus and herpes zoster $[6,25,29]$, places the elderly at greater risk and poses a challenge that require innovative solutions.

A key obstacle to finding such solutions is the fact that the frail elderly are often routinely excluded from clinical trials of new vaccines, which usually specify comorbidities, polypharmacy or dementia as exclusion criteria. This is compounded by the increased difficulty of recruiting older people into clinical trials because of cognitive impairment, longer recruitment processes (often requiring guardian consent), isolation, low mobility, difficulty attending follow up, altered body-mass index and potential lower renal and hepatic clearance of drugs and risk of adverse events, high mortality rates and attrition rates from trials (affecting statistical power) [30,31].

The systematic exclusion of frail elderly from RCTs results in a lack of evidence to inform immunization policy in the largest growing area of health need. This exclusion results in a vicious cycle of lack of evidence, lack of recommendation, and lack of protection for the oldest and frailest people in society, who have the highest burden of disease and risk of complications and death for all the major vaccine-preventable diseases of old age. The Shingles Prevention Study, for example, had fewer subjects aged $>79$ years compared to subjects aged $60-79$ years, with reduced statistical power to determine vaccine efficacy $>80$ years of age [32]. Yet the need for an evidence-base in the frail elderly will increase as the population continues to age, and there is an urgent need for more clinical trials in this group.

\section{EVIDENCE AROUND SPECIFIC VACCINES}

Influenza has a high burden of disease in the elderly and causes significant morbidity and mortality. Influenza vaccine is subject to seasonal variation in circulating virus match to vaccine, and within these limitations is shown to have acceptable efficacy in healthy adults, but not as much in the elderly [33]. A Cochrane Review which included only one randomized controlled trial of influenza vaccine concluded the vaccine has little or no efficacy in the elderly [34].

However, the challenge of doing a RCT of influenza vaccine efficacy is that firstly, such trials are subject to seasonal variation in influenza activity, and need to be run over several seasons to increase the probability of 
having enough circulating influenza; secondly that there is good vaccine match with circulating strains; thirdly, that follow up and laboratory testing of subjects for incident influenza is highly resource-intensive; and finally that the statistical power for showing efficacy against prevention of laboratory confirmed influenza requires trials of a scale that are unfeasible for independent researchers and can generally only be done by pharmaceutical companies. Trivalent Influenza vaccines are licensed on the basis of immunogenicity data, so there is no imperative for pharmaceutical companies to do such research. As such, most of the available data on influenza vaccine effectiveness come from observational epidemiologic studies and from outbreaks. Many of these studies do show effectiveness in the elderly, but each study is specific to the vaccine used at the time, the circulating influenza strains and the clinical outcome used in the study, which varies from death and hospitalization, influenza-like illness to laboratory confirmed infection [16,35-37].

A wide variation in vaccine effectiveness has been found in the elderly, but the extremely high burden of disease means that even a vaccine of lower effectiveness may have a population health impact on morbidity and mortality. Further, a vaccine which does not prevent influenza may still reduce the severity of infection and the risk of complications and death. A well conducted metaanalysis showed good effectiveness of pneumococcal polysaccharide vaccine against IPD, but not pneumonia, in the elderly [38] and yet there is a general lack of faith in this vaccine among providers. The prevailing lack of confidence in vaccines for the elderly filters down to affect the provider attitudes, which is one of the most powerful determinants of immunization $[39,40]$.

A paradigm shift would require acceptance of waning immunity with increasing age (along with lower vaccine effectiveness) as an immutable factor, and a shift of focus instead on solutions to this problem. Adjuvants have been explored as a means of improving influenza vaccine responses in the elderly [25], as has intradermal administration [41]. Both show some promise. Conjugate technology, developed to improve immune responses to children under the age of 2 years to polysaccharide vaccines, has been studied for elderly pneumococcal vaccination, with several studies comparing polysaccharide and conjugate vaccines in adults, but without a clear apparent benefit of one vaccine over the other [42]. However, a large RCT of 13-valent conjugate vaccine in adults is currently underway, powered to examine efficacy against pneumonia [43].

The herpes zoster vaccine contains the same Oka strain of vaccine virus used in the varicella vaccine but with over fourteen times the potency as the pediatric formulation [32]. Whilst this elicits good efficacy in the elderly within five year, efficacy declines with age and waning of vaccine immunity over time occurs [29]. In such a case, the exponentially rising burden of zoster with increasing age needs to be balanced with the reducing efficacy with age, rather than dismissing the vaccine entirely in the "older" elderly.

\section{THE NEED FOR A PARADIGM SHIFT FOR ELDERLY VACCINATION}

Elderly vaccination is too often viewed through the same lens as infant vaccination. For infant vaccination programs, we deal with an immature immune system which matures with age, especially with the waning of maternal antibodies by six months of age and the immature thymus in the first 6 months of life [44]. Infant vaccination programs are designed around a maturing immune system, knowing that with cumulative doses in a schedule, not only is immunological memory triggered by a second and third dose, but that the immune system of the infant becomes progressively more robust with the passing of time. With the elderly, however, we face a gradual decline in immune function, with consequent waning of vaccine induced immunity and decreasing vaccine effectiveness with increasing age [24].

A paradigm shift would involve changing the way we view elderly vaccination from viewing natural and progressive failure of the immune system as an insurmountable obstacle which makes vaccination pointless, to thinking of risk-benefit equations, the trade-off of burden of disease with vaccine effectiveness, and of creative and innovative strategies to boost immune responses in the elderly.

A vaccine which has hypothetically $70 \%$ efficacy in younger adults but only 50\% efficacy in older adults, might still have the population health impact on older adults if the disease burden is higher in older adults. For example, if there are 100,000 cases of a vaccine-preventable disease in a population among people aged 75 84 years, and 50,000 cases in people aged 65 - 74 years, then vaccinating the younger age group (70\% efficacy) will prevent 35,000 cases, but vaccinating the older age group with a vaccine that loses efficacy (50\% efficacy) would prevent 50,000 cases. The important vaccinepreventable diseases of the elderly such as influenza, pneumococcal disease and herpes zoster all have an increasing burden of disease with age, so that waning efficacy should be considered in the context of increasing disease incidence. A paradigm shift would require an assessment of the expected population health benefit of vaccines with lower effectiveness in the elderly, given higher burden of disease. This shift also requires the recognition that vaccinology is a predominantly pediatric discipline, with peak advisory bodies well represented in pediatrics and less well represented in adult medicine. As a result, the field of vaccinology has far fewer champions 
for adult immunization than it does for pediatric immunization. Further, adults represent the majority of the population, yet countries which have childhood vaccination registers have no adult vaccination registers, making immunization programs more challenging to monitor [45].

Unlike infants, adults are a mobile population with no easy access point for immunization, making them more difficult to target for scheduled vaccines. Some adults may not have contact with providers, or more commonly, the elderly may have multiple providers. The lack of universal health records or vaccination registers makes keeping track of vaccination in adults a challenge. It has been shown that doctor-recommendation predicts vaccination, even in people with negative perceptions of vaccination [40]. This means that the lack of provider confidence in elderly vaccination is a significant barrier.

\section{CONCLUSIONS}

In summary, developed countries are undergoing major demographic changes and ageing populations, yet our approach to immunization has not kept abreast of these changes. Protecting the elderly from the vaccine-preventable diseases is possible, and is not simply a function of the immune system and vaccine effectiveness. Whilst improved vaccine technologies must be developed to address the weakened elderly immune system, improving vaccination coverage and recording of adult vaccinations, addressing provider and patient barriers, and building an evidence-base of vaccine RCTS in the frail elderly are equally important. Other neglected areas include travel vaccination in the elderly, with a higher proportion of the elderly travelling after retirement [25]. Finally, cancerpreventing vaccines such as hepatitis B and Human papillomavirus vaccines have a long lead time from vaccination in childhood to prevention of cancer [46], and their benefits in adulthood are heavily discounted in standard cost-effectiveness analyses, which are biased against long-term benefits of vaccines [47].

Given the ageing of the population in high and middle income countries, it is time to view elderly vaccination through a different lens, to ensure the adequate representation on peak vaccination bodies with experts in geriatrics, to accept that vaccines are less immunogenic in the elderly, to not reject vaccines because they are less immunogenic in this age group, but to consider their population health impacts in the context of increased disease incidence, to view the glass as half full rather than half empty, and to make the most of vaccination as an available means of prevention in the elderly.

\section{REFERENCES}

[1] Lutz, W., Sanderson, W. and Scherbov, S. (2008) The coming acceleration of global population ageing. Nature,
451, 716-719. http://dx.doi.org/10.1038/nature06516

[2] Department of Economic and Social Affairs, Population Division. (2012) Population ageing and development. United Nations, New York.

[3] Australian Bureau of Statistics (2008) Future population growth and Aging, Population Projections, Australia, 2006 to 2101. Canberra.

http://www.abs.gov.au/Ausstats/abs@.nsf/mf/3222.0

[4] Humphreys, G. (2012) The health-care challenges posed by population ageing. Bulletin of the World Health Organization, 90, 82-83.

[5] Gavazzi, G. and Krause, KH. (2002) Ageing and infection. The Lancet Infectious Diseases, 2, 659-666. http://dx.doi.org/10.1016/S1473-3099(02)00437-1

[6] Ridda, I., Yin, J.K., King, C., MacIntyre, C.R. and McIntyre, P. (2012) The importance of pertussis in older adults: A growing case for reviewing vaccination strategy in the elderly. Vaccine, 30, 6745-6752.

http://dx.doi.org/10.1016/j.vaccine.2012.08.079

[7] Li, A., Newall, A.T., Britt, H. and Macintyre, C.R. (2012) The cost and disease burden of pneumonia in general practice in Australia. Vaccine, 30, 830-831. http://dx.doi.org/10.1016/j.vaccine.2011.11.103

[8] MacIntyre, C.R., Heywood, A.E., Kovoor, P., Ridda, I., Seale, H., Tan, T., et al. (2013) Ischaemic heart disease, influenza and influenza vaccination: A prospective case control study. Heart.

http://dx.doi.org/10.1136/heartjnl-2013-304320

[9] Warren-Gash, C., Smeeth, L. and Hayward, AC. (2009) Influenza as a trigger for acute myocardial infarction or death from cardiovascular disease: A systematic review. The Lancet Infectious Diseases, 9, 601-610. http://dx.doi.org/10.1016/S1473-3099(09)70233-6

[10] Corrales-Medina, V., Majid, M. and Musher, D. (2010) Role of acute infection in triggering acute coronary syndromes. The Lancet Infectious Diseases, 10, 83-92. http://dx.doi.org/10.1016/S1473-3099(09)70331-7

[11] Laughlin, L. (2010) Who’s minding the kids? Child care arrangements: Spring 2005 and Summer 2006. Current Population Reports, US Census Bureau, Washington DC, 2005, P70-P121.

[12] Vandell, D.L., McCartney, K., Owen, M.T., Booth, C. and Alison, C.-S. (2003) Variations in Child Care by Grandparents during the First Three Years. Journal of Marriage and Family, 65, 375-381. http://dx.doi.org/10.1111/j.1741-3737.2003.00375.x

[13] Booy, R., Lindley, R.I., Dwyer, D.E., Yin, J., Heron, L., et al. (2012) Treating and preventing influenza in aged care facilities: A cluster randomised controlled trial. PLoS One, 7, Article ID: e46509. http://dx.doi.org/10.1371/journal.pone.0046509

[14] Kirk, M.D., Moffatt, C.R., Hall, G.V., Becker, N., Booy, R., Heron, L., et al. (2010) The burden of infectious gastroenteritis in elderly residents and staff of long-term care facilities, Australia. Infection Control and Hospital Epidemiology, 31, 860-863.

http://dx.doi.org/10.1086/654000

[15] Seale, H., Weston, K.M., Dwyer, D.E., Zhu, M., Allchin, 
L., Booy, R., et al. (2009) The use of oseltamivir during an influenza B outbreak in a chronic care hospital. Influenza and Other Respiratory Viruses, 3, 15-20. http://dx.doi.org/10.1111/j.1750-2659.2008.00063.x

[16] Dean, A.S., Moffatt, C.R., Rosewell, A., Dwyer, D.E., Lindley, R.I., Booy, R., et al. (2010) Incompletely matched influenza vaccine still provides protection in frail elderly. Vaccine, 28, 864-867. http://dx.doi.org/10.1016/j.vaccine.2009.03.024

[17] Al-Murieb, A., Brown, A.M., Raulli, A., George, C., Gander, C., Forrester, P., et al. (2008) Evidence of pertussis clusters in three aged-care facilities in the former Macquarie Area Health Service, NSW. NSW Public Health Bulletin, 19, 157-160. http://dx.doi.org/10.1071/NB07054

[18] Mertens, P.L., Borsboom, G.J., Richardus and J.H. (2007) A pertussis outbreak associated with social isolation among elderly nuns in a convent. Clinical Infectious Diseases, 44, 266-268. http://dx.doi.org/10.1086/510428

[19] Ferson, M.J., Morgan, K., Robertson, P.W., Hampson, A.W., Carter, I. and Rawlinson, W.D. (2004) Concurrent summer influenza and pertussis outbreaks in a nursing home in Sydney, Australia. Infection Control and Hospital Epidemiology, 25, 962-966. http://dx.doi.org/10.1086/502327

[20] Mertens, P.L., Stals, F.S., Schellekens, J.F., Houben, A.W. and Huisman, J. (1999) An epidemic of pertussis among elderly people in a religious institution in The Netherlands. European Journal of Clinical Microbiology \& Infectious Diseases, 18, 242-247. http://dx.doi.org/10.1007/s100960050271

[21] Addiss, D.G., Davis, J.P., Meade, B.D., Burstyn, D.G., Meissner, M., Zastrow, J.A., et al. (1991) A pertussis outbreak in a Wisconsin nursing home. The Journal of Infectious Diseases, 164, 704-710. http://dx.doi.org/10.1093/infdis/164.4.704

[22] Ridda, I., MacIntyre, C.R., Lindley, R.I., McIntyre, P.B., Sullivan, J., Gilbert, G., et al. (2007) Predictors of pneumococcal vaccination uptake in hospitalized patients aged 65 years and over shortly following the commencement of a publicly funded national pneumococcal vaccination program in Australia. Human Vaccines, 3, 83-86. http://dx.doi.org/10.4161/hv.3.3.3925

[23] Panda, A., Arjona, A., Sapey, E., Bai, F., Fikrig, E., Montgomery, R.R., et al. (2009) Human innate immunosenescence: Causes and consequences for immunity in old age. Trends in Immunology, 30, 325-333. http://dx.doi.org/10.1016/j.it.2009.05.004

[24] Weinberger, B., Herndler-Brandstetter, D., Schwanninger, A., Weiskopf, D. and Grubeck-Loebenstein, B. (2008) Biology of immune responses to vaccines in elderly persons. Clinical Infectious Diseases, 46, 1078-1084. http://dx.doi.org/10.1086/529197

[25] Weinberger, B. and Grubeck-Loebenstein, B. (2012) Vaccines for the elderly. Clinical Microbiology and Infection, 18, 100-108. http://dx.doi.org/10.1111/j.1469-0691.2012.03944.x

[26] Derhovanessian, E. and Pawelec, G. (2012) Vaccination in the elderly. Microbial Biotechnology, 5, 226-232. http://dx.doi.org/10.1111/j.1751-7915.2011.00283.x

[27] Insinga, R.P., Itzler, R.F., Pellissier, J.M., Saddier, P. and Nikas, A.A. (2005) The incidence of herpes zoster in a United States administrative database. Journal of General Internal Medicine, 20, 748-753. http://dx.doi.org/10.1111/j.1525-1497.2005.0150.x

[28] Hope-Simpson, R.E. (1965) The nature of herpes zoster: A long-term study and a new hypothesis. Proceedings of Royal Society of Medicine, 58, 9-20.

[29] Schmader, K.E., Oxman, M.N., Levin, M.J., Johnson, G., Zhang, J.H., Betts, R., et al. (2012) Persistence of the efficacy of zoster vaccine in the shingles prevention study and the short-term persistence substudy. Clinical Infectious Diseases, 55, 1320-1328. http://dx.doi.org/10.1093/cid/cis638

[30] Ridda, I., MacIntyre, C.R., Lindley, R.I. and Tan, T.C. (2010) Difficulties in recruiting older people in clinical trials: An examination of barriers and solutions. Vaccine, 28, 901-906. http://dx.doi.org/10.1016/j.vaccine.2009.10.081

[31] Ridda, I., Lindley, R. and MacIntyre, C.R. (2008) The challenges of clinical trials in the exclusion zone: The case of the frail elderly. Australasian Journal on Ageing, 27, 61-66. http://dx.doi.org/10.1111/j.1741-6612.2008.00288.x

[32] Oxman, M.N., Levin, M.J., Johnson, G.R., Schmader, K.E., Straus, S.E., Gelb, L.D., et al. (2005) A vaccine to prevent herpes zoster and postherpetic neuralgia in older adults. The New England Journal of Medicine, 352, 22712284. http://dx.doi.org/10.1056/NEJMoa051016

[33] Osterholm, M.T., Kelley, N.S., Sommer, A. and Belongia, E.A. (2012) Efficacy and effectiveness of influenza vaccines: A systematic review and meta-analysis. The Lancet Infectious Diseases, 12, 36-44.

http://dx.doi.org/10.1016/S1473-3099(11)70295-X

[34] Jefferson, T., Di Pietrantonj, C., Al-Ansary, L.A., Ferroni, E., Thorning, S. and Thomas, R.E. (2010) Vaccines for preventing influenza in the elderly. Cochrane Database of Systematic Reviews, 17, Article ID: CD004876.

[35] Talbot, H.K., Griffin, M.R., Chen, Q., Zhu, Y., Williams, J.V., Edwards, K.M. (2011) Effectiveness of seasonal vaccine in preventing confirmed influenza-associated hospitalizations in community dwelling older adults. The Journal of Infectious Diseases, 203, 500-508. http://dx.doi.org/10.1093/infdis/jiq076

[36] Kissling, E., Valenciano, M., Cohen, J.M., Oroszi, B., Barret, A-S., Rizzo, C., et al. (2011) I-MOVE multi-centre case control study 2010-11: Overall and stratified estimates of influenza vaccine effectiveness in Europe. PLoS ONE, 6, Article ID: e27622. http://dx.doi.org/10.1371/journal.pone.0027622

[37] Hara, M., Sakamoto, T. and Tanaka, K. (2008) Influenza vaccine effectiveness among elderly persons living in the community during the 2003-2004 season. Vaccine, 26, 6477-6480. http://dx.doi.org/10.1016/j.vaccine.2008.06.035

[38] Moberley, S.A., Holden, J., Tatham, D.P. and Andrews, R.M. (2013) Vaccines for preventing pneumococcal infection in adults. Cochrane Database Systematic Reviews, 
23, Article ID: CD000422.

[39] Ridda, I., Lindley, R. and MacIntyre, C.R. (2009) Differences in the attitude and knowledge of hospital health care workers regarding older patient vaccinations. Human Vaccines, 5, 761-764. http://dx.doi.org/10.4161/hv.5.11.9869

[40] Ridda, I., Motbey, C., Lam, L., Lindley, R., McIntyre, P.B. and MacIntyre, C.R. (2008) Factors associated with Pneumococcal immunization among hospitalised elderly persons: A survey of patient's perception, attitude, and knowledge. Vaccine, 26, 234-240.

http://dx.doi.org/10.1016/j.vaccine.2007.10.067

[41] Falsey, A.R. (2010) New emerging technologies and the intradermal route: The novel way to immunize against influenza. Vaccine, 28, D24-D32.

http://dx.doi.org/10.1016/j.vaccine.2010.08.026

[42] O’Brien, K.L. (2009) Pneumococcal conjugate vaccine, polysaccharide vaccine, or both for adults? We're not there yet. Clinical Infectious Disease, 49, 1326-1328. http://dx.doi.org/10.1086/606047

[43] Hak, E., Grobbee, D.E., Sanders, E.A., Verheij, T.J., Bolkenbaas, M., Huijts, S.M., Gruber, W.C., Tansey, S., McDonough, A., Thoma, B., Patterson, S., van Alphen, A.J. and Bonten, M.J. (2008) Rationale and design of CAPITA:
A RCT of 13-valent conjugated pneumococcal vaccine efficacy among older adults. Netherlands Journal of Medicine, 66, 378-383.

[44] PrabhuDas, M., Adkins, B., Gans, H., King, C., Levy, O., Ramilo, O. and Siegrist, C.A. (2011) Challenges in infant immunity: Implications for responses to infection and vaccines. Nature Immunology, 12, 189-194. http://dx.doi.org/10.1038/ni0311-189

[45] Hull, B.P., Deeks, S.L. and McIntyre, P.B. (2009) The Australian childhood immunisation register-A model for universal immunisation registers? Vaccine, 27, 5054-5060. http://dx.doi.org/10.1016/j.vaccine.2009.06.056

[46] Chang, M.H., You, S.L., Chen, C.J., Liu, C.J., Lee, C.M., Lin, S.M., Chu, H.C., Wu, T.C., Yang, S.S., Kuo, H.S., Chen, D.S. and the Taiwan Hepatoma Study Group (2009) Decreased incidence of hepatocellular carcinoma in heaptitis B vaccinees: A 20-year follow-up study. Journal of the National Cancer Institute, 101, 1348-1355. http://dx.doi.org/10.1093/jnci/djp288

[47] Beutels, P., Scuffham, P.A. and MacIntyre, C.R. (2008) Funding of drugs: Do vaccines arrant a different approach? Lancet Infectious Diseases, 8, 727-733. http://dx.doi.org/10.1016/S1473-3099(08)70258-5 